\title{
Session 3: Cancer - II
}

Wednesday 6 October 2004. Moderators: H. Peter Vollmers and Mark C. Glassy

\author{
[11.30-12.00] \\ [Plenary Lecture] \\ Mark C. Glassy \\ Rajko Medinica Research Foundation, Shantha West, \\ UCSD, USA
}

Abstract not received.

\section{[12.00-12.20]}

CFR-1 as a new diagnostic tool for prostate cancer precursors defined by the human monoclonal IgM antibody PAM-1

Stephanie Brändlein, Philipp Ströbel, Ewa Wozniak, Hans Konrad Müller-Hermelink and H. Peter Vollmers*

Institute of Pathology, University of Würzburg, Josef-Schneider-Str. 2, D-97080 Würzburg, Germany *Corresponding author. E-mail: peter.vollmers@ mail.uni-wuerzburg.de

Abstract: Precancerous epithelial lesions are sites of uncontrolled cellular proliferation, generated by irreversible genetic changes. Not all these lesions progress to invasive cancer, some may even regress, but early detection of abnormal cells can be crucial for survival of the patient. Prostate cancer is still diagnosed by pathologists based on subjective assessment of altered cell and tissue structure. Enlargement of the nucleolus is the only key diagnostic feature of high-grade prostatic intraepithelial neoplasia (PIN), an early stage that appears to be the precursor to the majority of invasive prostate cancers, but its status as a neoplastic precursor remains only putative. There is clearly a need for additional diagnostic tools, but conventional immunohistochemical markers like Ki-67, PSA or p53 do not really help to differentiate between benign and malignant tissue at an early stage. They are not tumor-specific or only expressed on a subset of PINs and are therefore not predictive. The fully human monoclonal IgM an- tibody PAM-1 was isolated from a patient with stomach carcinoma and binds to a new variant of the posttranscriptionally modified receptor CFR- 1 . This receptor is expressed on nearly all epithelial cancers of every type and origin, but not on healthy tissue. CFR-1 is also present on precursor lesions found in: Helicobacter pylori-induced gastritis, intestinal metaplasia and dysplasia of the stomach, ulcerative colitis-related dysplasia and adenomas of the colon, Barrett metaplasia and dysplasia of the esophagus, squamous cell metaplasia and dysplasia of the lung and cervical intraepithelial neoplasia. We present new data on the unique expression of this new CFR-1 receptor on PINs, what makes the PAM-1 antibody an ideal reliable diagnostic tool for the detection of precancerous and cancerous lesions.

\section{[12.20-12.40]}

Expression and production of biologically active anti-tumoral antibodies in transgenic plant systems Iann Rancé ${ }^{1, *}$, Gregg Wallis ${ }^{1, *}$, Virginie Jonval ${ }^{1}$, Hélène Laparra ${ }^{1}$, Nathalie Duchateau ${ }^{1}$, Daniel Burtin $^{1}$, Mike McKnight ${ }^{2}$ and Mark Glassy ${ }^{2}$ ${ }^{1}$ MERISTEM Therapeutics, 8 Rue des frères Lumière, 63100 Clermont-Ferrand, France

${ }^{2}$ Shantha West Inc. 11211 Sorento Valley Road, Suite C, SanDiego, CA-92121, USA

*Corresponding authors. E-mail: \{irance,gwallis\}@ meristem-therapeutics.com

To ascertain the effect of an oligoclonal strategy to cure tumor growth in several cancer pathologies, a set of 4 well characterized antitumoral IgA monoclonal antibodies were isolated, and expressed in $\mathrm{CHO}$ cells. $\mathrm{Pu}-$ rification of these antibodies at small scale was successful but at ineffective levels to undertake clinical studies. The production of antibodies with high bioactivity in stable, cost effective, large scale homogeneous batches presents a number of manufacturing challenges. The decision was made to try a feasibility study of ex- 
pression of these four antibodies in Corn plants, using MERISTEM ${ }^{\circledR}$ Therapeutics' unique proprietary plant engineering concept, Molecular Pharming ${ }^{\circledR}$. The goal of the study was to demonstrate the feasibility of expression of a bioactive antibody in a single plant and produce developmental scale levels of each molecule.

For each antibody the light and heavy chains were "vegetalized" and fused to a plant secretion signal peptide. Both sequences were cloned in our proprietary expression vector allowing high transcription and translation of both chain expression cassettes. Expression was specifically targeted to the endosperm of the corn mature seeds. Vector born T-DNAs were transfered to the plant genome via use of an hypervirulent strain of the plant pathogenic bacteria Agrobacterium tumefaciens.

Forty stably transformed Corn plants were regenerated after in vitro culture steps and subsequently brought to soil in a greenhouse. A number of genetic studies was conducted in order to characterize the insertion borders and the copy number of the transgenes in each transgenic T0 plant. Only the plants having a single copy insertion with no extra T-DNA borders were kept. Biochemical analysis of the various transformants allowed the selection of the highest expressors. Expression levels ranged between 0.01 to up to $4.8 \%$ of the seed total soluble proteins (TSP) in corn. Western analysis confirmed the presence of the intact tetramer.

Plants expressing the antibody molecule at a level over $0.5 \%$ TSP were pollinated and propagated to the next generation. At each generation, genetic and biochemical analysis were conducted so as to check for inheritance of the antibody expression cassettes and expression yields. Starting at generation T3, the transgenic biomass (under the form of dry seeds) was used to develop a purification scheme at the laboratory scale.

Our data demonstrate that using our technology platform clinical grade batches of purified recombinant antibody can be produced, in a animal free system, without the need of an expensive cell culture facility.

\section{[12.40-13.00]}

\section{Altering distribution and pharmacokinetics of a fab through association with albumin} Mark S. Dennis, Debra L. Dugger, Joe Kowalski, Simon Williams, Min Zhang, Paul McDonald, Allen Nguyen, Arthur Reyes, Wai Lee Wong, Lisa A. Damico and Ralph H. Schwall Genentech, Inc., 1 DNA Way, South San Francisco, CA 94080, USA

Tel.: +1 650225 1162; E-mail: msd@gene.com
We have investigated association with albumin as a means to improve the distribution and pharmacokinetic properties of a Fab derived from Herceptin and directed to the tumor antigen HER2 (erbB2). Phagederived peptides that specifically bind serum albumin with high affinity, when fused to the Fab, create an albumin-binding Fab (AB.Fab) capable of binding both antigen and albumin [1].

Interaction with albumin resulted in a reduced in vivo clearance of the Fab that could be modified by altering the affinity for albumin (Fig. 1). The halflife was extended up to 37 -fold in rabbit exceeding that of a Fab'2 and comparable to PEG conjugated Fab molecules, immunoadhesins, and albumin fusions. However, this improved pharmacokinetic behavior of the AB.Fab was achieved without a dramatic increase in the size of the Fab.

Like IgG, albumin has recently been shown to utilize the major histocompatability complex-related Fc recpetor $(\mathrm{FcRn})$ to survive pinocytosis and extend its half-life in vivo [2]. A pH titration of the albuminbinding peptide (AB.peptide) binding to albumin suggests that this interaction is stable at $\mathrm{pH} 6$ (Fig. 2). In addition, this peptide did not interfere with the ability of albumin to bind FcRn (Fig. 3), thus it is reasonable to expect that the AB.Fab is capable of surviving pinocytosis in vivo. The ability to be recycled via the FcRn receptor and reduced renal clearance as a result of an increase in apparent molecular size when in complex

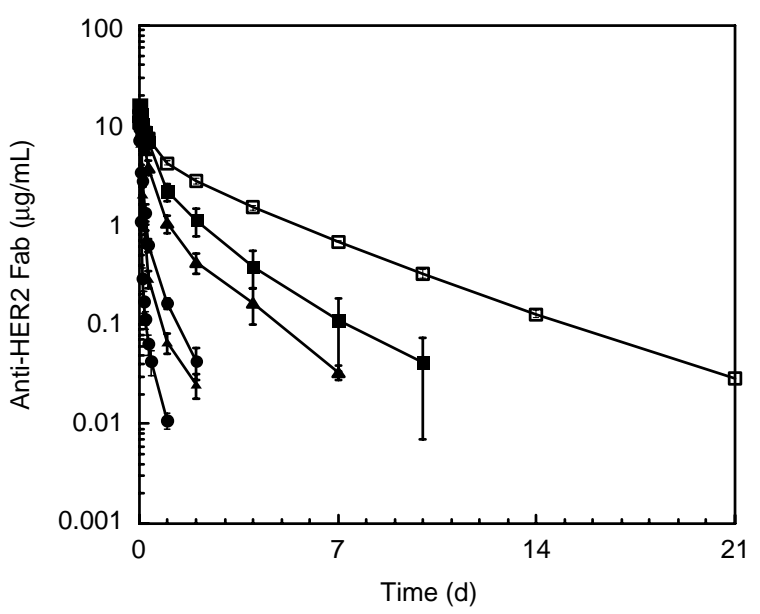

Fig. 1. The pharmacokinetic profile in rabbit of $A B$.Fab variants with various binding affinities for rabbit albumin. Groups of three New Zealand White rabbits were given an intravenous bolus of $0.5 \mathrm{mg} / \mathrm{kg}$ AB.Fab4D5 variants $\mathrm{H}(\square), \mathrm{H} 8(\boldsymbol{\square}), \mathrm{H} 4(\boldsymbol{\Delta}), \mathrm{H} 10(\bigcirc), \mathrm{H} 11$ (•) or Fab4D5 $(\bullet)$. The affinities of these molecules for rabbit albumin are $42 \mathrm{nM}, 210 \mathrm{nM}, 460 \mathrm{nM}, 1.25 \mu \mathrm{M},>10 \mu \mathrm{M}$ and $>50 \mu \mathrm{M}$, respectively. 


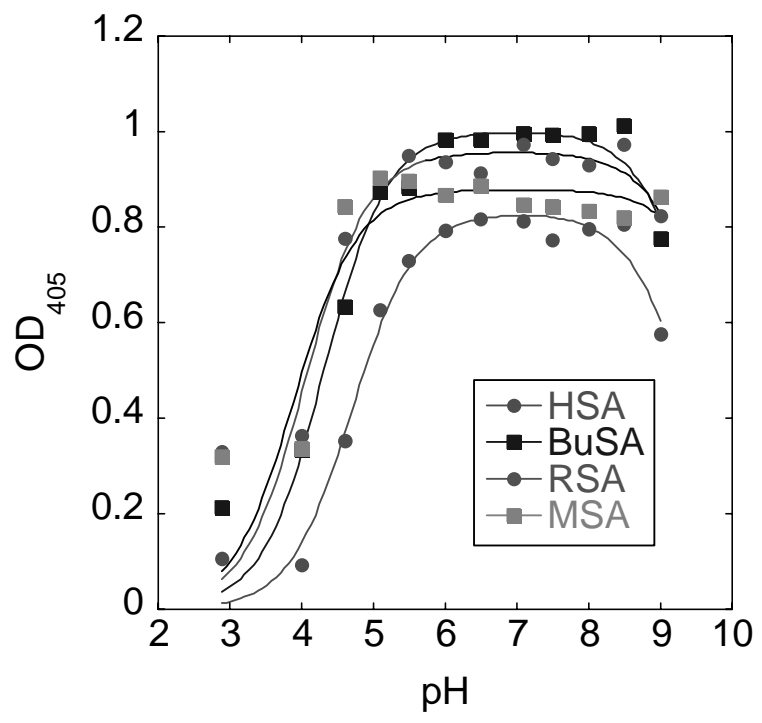

Fig. 2. $\mathrm{pH}$ dependent binding of the AB.peptide to albumin. The binding of phage bearing the AB.peptide to human (HSA), rabbit (BuSA), rat (RSA), or mouse (MSA) albumin was measured as a function of $\mathrm{pH}$.

with albumin likely both contribute to the observed extended half-life of the AB.Fab.

The potential benefit of using an AB.Fab for tumor targeting was investigated by comparing the tumor deposition of fluorescently labeled Fab, AB.Fab and IgG.
In a mouse xenograft model containing HER2 overexpressing tumors, significant tumor deposition was observed at $2 \mathrm{hr}$ for the Fab, but not until $24 \mathrm{~h}$ for the IgG (Figs 4 and 5). At 24 h, the Fab was completely absent in the tumor and observed only in the kidney. In contrast the AB.Fab was observed early ( $2 \mathrm{~h})$ in the tumor and its presence was sustained beyond $24 \mathrm{~h}$, similar to the IgG. Thus the Ab.Fab targets the tumor more rapidly than the IgG and its presence is sustained beyond that of the Fab. Rapid and specific tumor penetration by the AB.Fab was accompanied by excellent tumor retention and low normal tissue backgrounds. These properties make AB.Fabs excellent tumor targeting agents for imaging and cancer therapy.

\section{References}

[1] M.S. Dennis, M. Zhang, Y.G. Meng, M. Kadkhodayan, D. Kirchhofer, D. Combs and L.A. Damico, Albumin binding as a general stategy for improving the pharmacokinetics of proteins, J. Biol. Chem. 277 (2002), 35035.

[2] C. Chaudhury, S. Mehnaz, J.M. Robinson, W.L. Hayton, D.K. Pearl, D.C. Roopenian and C.L. Anderson, The major histocompatibility complex-related Fc receptor for IgG (FcRn) binds albumin and prolongs its lifespan, J. Exp. Med. 197 (2003), 315.

[3] M. Birchler, G. Neri, L. Tarli, C. Halin, F. Viti and D. Neri, Infrared photodetection for the in vivo localisation of phagederived antibodies directed against angiogenic markers, $J$. Immunol. Methods 231 (1999), 239. 